\title{
Burgers Denkleminin Sayısal Çözümleri İçin Logaritmik Sonlu Fark Yöntemleri
}

\author{
Gonca ÇELIKKTEN*
}

Kafkas Üniversitesi, Fen Edebiyat Fakültesi, Matematik Bölümü, 36100, Kars, Türkiye

Geliş / Received: 12/02/2020, Kabul / Accepted: 15/09/2020

\section{Öz}

Bu çalışmada, Burgers denkleminin sayısal çözümlerini elde etmek için kapalı ve tamamen kapalı logaritmik sonlu fark yöntemleri kullanılmıştır. Yöntemlerin performansını test etmek için iki model problem kullanılmıştır. Tam çözümlerin ve diğer birkaç yöntemle elde edilen sayısal çözümlerin karşılaştırılmaları tablolarla birlikte verilmiştir. Sonuçların doğruluğunu göstermek için $L_{2}$ ve $L_{\infty}$ hata normları kullanılmıştır.

Anahtar Kelimeler: Burgers denklemi, kapalı logaritmik sonlu fark yöntemi, tamamen kapalı logaritmik sonlu fark yöntemi, von- Neumann kararlılık yöntemi.

\section{Logarithmic Finite Difference Methods for Numerical Solutions of Burgers Equation}

\begin{abstract}
In this study, implicit and fully implicit logarithmic finite difference methods have been used to obtain the numerical solutions of Burgers equation. Two model problems have been used to test the performance of the methods. Comparisons of the exact solutions and the numerical solutions obtained by several other methods have been present with tables. $L_{2}$ and $L_{\infty}$ error norms have been used to demonstrate the correctness of the results.
\end{abstract}

Keywords: Burgers equation, implicit logarithmic finite difference method, fully implicit logarithmic finite difference method, von- Neumann stability method. 


\section{Giriş}

$$
\frac{\partial U}{\partial t}+U \frac{\partial U}{\partial x}=v \frac{\partial^{2} U}{\partial x^{2}}, \quad a<x<b, \quad t>0
$$

ile verilen Burgers denklemini

$U(x, 0)=f(x), \quad a<x<b$

başlangıç şartı ve

$U(a, t)=g_{1}(t), \quad U(b, t)=g_{2}(t), \quad t>0$

sınır şartları ile göz önüne alalım. Burada $f(x), g_{1}(t)$ ve $g_{2}(t)$ bilinen fonksiyonlar ve $v>0$ akışkanın kinematik viskozitesi olarak bilinen parametredir.

Burgers denklemi ilk önce Bateman (1915) tarafindan bir makalede sunuldu ve daha sonra Burgers (1939) bu denklemi türbülans modeli olarak kullandı. Literatürde birkaç yazar tarafından denklemin sayısal çözümlerini elde etmek için farklı yöntem ve teknikler kullanılmıştır (Aksan ve Özdeş, 2004; Aksan, 2005; Çelikten vd., 2017; Gülsu ve Öziş, 2005; Gülsu, 2006; İnan ve Bahadır, 2013a ve b; Kutluay vd., 2004; Öziş vd., 2003; Salkuyeh ve Sharafeh, 2009; Korkmaz ve Dag, 2011; Zhang ve Wang, 2012; Mittal ve Jain, 2012; Jiwari, 2012). Çelikten vd. (2017) açık logaritmik sonlu fark yaklaşımını kullanarak denklemin sayısal çözümlerini elde etmiştirler. Gülsu ve Öziş (2005) denklemin sayısal çözümlerini elde etmek için klasik açık sonlu fark yöntemine kısıtlayıcı Taylor yaklaşımını uyguladılar. Gülsu (2006) denklemin sayısal çözümlerini elde etmek için klasik kapalı sonlu fark yöntemine kısıtlayıcı Pade yaklaşımını uyguladı. İnan ve Bahadır (2013a ve b) denklemin sayısal çözümlerini elde etmek için kapalı, tamamen kapalı ve açık üstel sonlu fark şemalarını kullanmıştırlar. Kutluay vd. (2004) tek boyutlu Burgers benzeri denklemlerin sayısal çözümlerini hesaplamak için en küçük kareler içeren ikinci dereceden bir B-spline sonlu elemanlar yöntemi kullandilar. Burgers denklemi Salkuyeh ve Sharafeh (2009) tarafindan denkleme Hopf-Cole dönüşümü uygulanmış ve daha sonra ortaya çıkan isı denkleminin konum değişkenine göre ayrıştırılmasıyla denklem sayısal olarak çözülmüştür.

Bu çalışmada, Burgers denkleminin sayısal çözümlerini elde etmek için kapalı ve tamamen kapalı logaritmik sonlu fark yöntemleri kullanılmıştır. Von-Neumann kararlılık yöntemi kullanılarak kapalı ve tamamen kapalı logaritmik sonlu fark yöntemlerinin şartsız kararlı olduğu gösterilmiştir. Yöntemlerin performansını test etmek için iki model problem kullanılmıştır.

\section{Materyal ve Metot}

\subsection{Model problemler}

\section{Problem 1:}

İlk olarak (1) ile verilen Burgers denklemi

$$
U(x, 0)=\sin (\pi, x), 0<x<1
$$

başlangıç şartı ve

$$
U(0, t)=U(1, t)=0, \quad t>0 .
$$

sınır şartları ile ele alındı. Bu problemin tam çözümü 


$$
U(x, t)=\frac{2 \pi v \sum_{n=1}^{\infty} b_{n} \exp \left(-n^{2} \pi^{2} v t\right) n \sin (n \pi x)}{b_{0}+\sum_{n=1}^{\infty} b_{n} \exp \left(-n^{2} \pi^{2} v t\right) \cos (n \pi x)}
$$

olup, burada $a_{0}$ ve $a_{n}$ katsayıları

$$
\begin{aligned}
& b_{0}=\int_{0}^{1} \exp \left[-(2 \pi v)^{-1}(1-\cos (\pi x))\right] d x \\
& b_{n}=2 \int_{0}^{1} \exp \left[-(2 \pi v)^{-1}(1-\cos (\pi x))\right] \cos (n \pi x) d x, \\
& n=1,2,3, \ldots
\end{aligned}
$$

şeklindedir.

\section{Problem 2:}

(1) ile verilen Burgers denklemi

$$
U(x, 0)=4 x(1-x), 0<x<1
$$

başlangıç şartı ve

$$
U(0, t)=U(1, t)=0, \quad t>0 .
$$

sınır şartları ile ele alındı. Bu problemin tam çözümü

$$
U(x, t)=\frac{2 \pi v \sum_{n=1}^{\infty} a_{n} \exp \left(-n^{2} \pi^{2} v t\right) n \sin (n \pi x)}{a_{0}+\sum_{n=1}^{\infty} a_{n} \exp \left(-n^{2} \pi^{2} v t\right) \cos (n \pi x)}
$$

olup, burada $a_{0}$ ve $a_{n}$ katsayıları

$$
\begin{aligned}
& b_{0}=\int_{0}^{1} \exp \left[-x^{2}(3 v)^{-1}(3-2 x)\right] d x \\
& b_{n}=2 \int_{0}^{1} \exp \left[-x^{2}(3 v)^{-1}(3-2 x)\right] \cos (n \pi x) d x, \\
& n=1,2,3, \ldots
\end{aligned}
$$

şeklindedir.

\subsection{Sayısal yöntem}

$U(x, t) \operatorname{nin}\left(x_{i}, t_{n}\right)$ düğüm noktasındaki sonlu fark yaklaşımını $u_{i}^{n}$ ile gösterelim, burada $N$ bölüntü sayısı olmak üzere $x_{i}=i h(i=0,1, \ldots, N), \quad t_{n}=t_{0}+n k(n=0,1,2, \ldots)$, $h=\frac{b-a}{N} \quad x$-yönündeki bölüntü uzunluğu ve $k$ zaman adımıdır.

(1) denklemini düzenlersek

$$
\frac{\partial U}{\partial t}=-U \frac{\partial U}{\partial x}+v \frac{\partial^{2} U}{\partial x^{2}}
$$

(6)

elde edilir. (6) denklemini $\frac{\partial F}{\partial U}$ ile çarparak aşağıdaki denklemi elde ederiz:

$$
\frac{\partial F}{\partial U} \frac{\partial U}{\partial t}=F^{\prime}(U)\left(-U \frac{\partial U}{\partial x}+v \frac{\partial^{2} U}{\partial x^{2}}\right)
$$

ve

$$
\frac{\partial F}{\partial t}=F^{\prime}(U)\left(-U \frac{\partial U}{\partial x}+v \frac{\partial^{2} U}{\partial x^{2}}\right) .
$$

Burada $F(U)$ herhangi bir sürekli türevlenebilir fonksiyondur. $\frac{\partial F}{\partial t}$ türevi yerine geri fark yaklaşımını kullanırsak (8) denkleminin sonlu fark yaklaşımı aşağıdaki gibi elde edilir:

$$
F\left(U_{i}^{n+1}\right)=F\left(U_{i}^{n}\right)+k F^{\prime}(U)\left(-U \frac{\partial U}{\partial x}+v \frac{\partial^{2} U}{\partial x^{2}}\right) .
$$


$F(U)=e^{U} \quad$ olsun, bu durumda (9) denklemindeki türevler için sonlu fark yaklaşımları kullanılarak sırasıyla aşağıdaki kapalı logaritmik sonlu fark yaklaşımı (K-LSFY) ve tamamen kapalı logaritmik sonlu fark yaklaşımı (TKLSFY) elde edilir:

$\mathrm{K}-\mathrm{LSFY} \quad U_{i}^{n+1}=U_{i}^{n}+\ln \left\{1-\frac{k}{2 h} U_{i}^{n}\left(U_{i+1}^{n+1}-U_{i-1}^{n+1}\right)+r v\left(U_{i+1}^{n+1}-2 U_{i}^{n+1}+U_{i-1}^{n+1}\right)\right\}$

TK-LSFY

$$
U_{i}^{n+1}=U_{i}^{n}+\ln \left\{1-\frac{k}{2 h} U_{i}^{n+1}\left(U_{i+1}^{n+1}-U_{i-1}^{n+1}\right)+r v\left(U_{i+1}^{n+1}-2 U_{i}^{n+1}+U_{i-1}^{n+1}\right)\right\}
$$

burada $r=\frac{k}{h^{2}}, 1 \leq \mathrm{i} \leq \mathrm{N}-1$ dir.

(10) ve (11) denklemleri lineer olmayan fark denklemlerinin sistemleridir. Bu lineer olmayan denklem sistemlerinin

$$
G(V)=0
$$

formunda olduğunu kabul edelim, burada $G=\left[g_{1}, g_{2}, \ldots, g_{N-1}\right]^{T}$ ve $V=\left[U_{1}^{n+1}, U_{2}^{n+1}, \ldots, U_{N-1}^{n+1}\right]^{T}$ şeklindedir.

sistemini Newton Yöntemi ile çözmek için aşağıdaki adımlar izlenir:

1) $V^{(0)}$ başlangıç değerlerinin belirlenmesi

2) $m=0,1,2, \ldots$ için

$$
J\left(V^{(m)}\right) \delta^{(m)}=-G\left(V^{(m)}\right) \quad \text { sisteminin }
$$

çözümü ve

$V^{(m+1)}=V^{(m)}+\delta^{(m)}$ hesaplanması.

Burada $J\left(V^{(m)}\right)$ analitik olarak hesaplanan Jacobian matrisidir. Her bir zaman adımında Newton yönteminin durdurma kriteri $\left\|G\left(V^{(m)}\right)\right\| \leq 10^{-5}$ olarak alınmıştır (İnan ve Bahadır, 2013a).

\section{Kararlılık Analizi}

şeklinde yazılabilir. (13) ve (14) yaklaşımlarının kararlılık analizi için vonNeumann yöntemi kullanılacaktır. VonNeumann yöntemi sadece lineer fark denklemlerine uygulandığından (13) ve (14) yaklaşımlarının kararlılığını incelemek için sırasıyla yaklaşımlardaki $U_{i}^{n}$ ve $U_{i}^{n+1}$ değişkenleri sabitmiş gibi kabul edilerek lineer olmayan terimler lineerleştirilir. 
$U_{i}^{n}=U_{i}^{n+1}=\hat{U}=$ sabit olarak ele alınırsa, bu durumda (13) ve (14) yaklaşımlarının lineer formu

$$
\begin{aligned}
U_{i}^{n+1} & =U_{i}^{n}-\frac{k}{2 h} \hat{U}\left(U_{i+1}^{n+1}-U_{i-1}^{n+1}\right) \\
& +r v\left(U_{i+1}^{n+1}-2 U_{i}^{n+1}+U_{i-1}^{n+1}\right)
\end{aligned}
$$

şeklinde yazılabilir. Bu yaklaşıma vonNeumann kararlılık yöntemini uygulayalım. (15) yaklaşımında $U_{i}^{n}$ yerine

$U_{i}^{n}=\gamma^{n} e^{I \beta i h}, I=\sqrt{-1}$

yazılır ve gerekli düzenlemeler yapılırsa güçlendirme faktörü

$$
\gamma=\frac{1}{1+4 r v \sin ^{2} \frac{\beta h}{2}+i \frac{k \hat{U}}{h} \sin \beta h}
$$

olarak elde edilir. von-Neumann yönteminde kararlılık şartı $|\gamma| \leq 1$ olup $\mathrm{Bu}$ şart her $r>0$ için sağlanacağından yaklaşımlar şartsız kararlıdır.

\section{Bulgular}

Kapalı ve tamamen kapalı logaritmik sonlu fark yöntemleri kullanılarak ele alınan model problemlerin sayısal çözümleri elde edilmiştir.

$$
\begin{aligned}
& L_{2}=\left\|U-u_{N}\right\|_{2}=\sqrt{h \sum_{j=0}^{N}\left|U_{j}-\left(u_{N}\right)_{j}\right|^{2}}, \\
& L_{\infty}=\left\|U-u_{N}\right\|_{\infty}=\max _{j}\left|U_{j}-\left(u_{N}\right)_{j}\right|
\end{aligned}
$$

şeklinde tanımlanan $L_{2}$ ve $L_{\infty}$ hata normları kullanılarak sonuçların doğruluğu gösterilmiştir, burada $U$ ve $u_{N}$ sirasıyla tam çözümleri ve hesaplanan sayısal çözümleri göstermektedir.

Problem 1 için sunulan yöntemler ile elde edilen sayısal sonuçlar Tablo 1-5 ile gösterilmektedir. Tablo 1 ile $k=10^{-5}$, $v=1$ ve $N^{\prime}$ nin farklı değerleri için $t=0.1$ zamanında K-LSFY kullanılarak elde edilen sayısal sonuçlar sunulmuştur. Tablo 2 ile $k=10^{-5}, v=1$ ve $N$ ' nin farklı değerleri için $t=0.1$ zamanında TK-LSFY kullanılarak elde edilen sayısal sonuçlar sunulmuştur. Tablo 1 ve Tablo 2 den $N^{\prime}$ nin değeri büyüdükçe yani konum için bölüntü sayısı arttıkça $L_{2}$ ve $L_{\infty}$ hata normlarının değerlerinin azaldığ görülmektedir.

Tablo 1. Problem 1 için K-LSFY ile elde edilen çözümler ile tam çözümlerin karşılaştırılması

\begin{tabular}{|l|l|l|l|l|l|}
\hline$x$ & $\begin{array}{l}\text { Tam } \\
\text { Çözüm }\end{array}$ & $N=100$ & $N=80$ & $N=40$ & $N=20$ \\
\hline 0.1 & 0.109538 & 0.109548 & 0.109552 & 0.109588 & 0.109729 \\
\hline 0.2 & 0.209792 & 0.209811 & 0.209820 & 0.209889 & 0.210169 \\
\hline 0.3 & 0.291896 & 0.291924 & 0.291936 & 0.292037 & 0.292443 \\
\hline 0.4 & 0.347924 & 0.347958 & 0.347974 & 0.348101 & 0.348611 \\
\hline 0.5 & 0.371577 & 0.371616 & 0.371634 & 0.371778 & 0.372356 \\
\hline 0.6 & 0.359046 & 0.359085 & 0.359103 & 0.359251 & 0.359844 \\
\hline
\end{tabular}




\begin{tabular}{|l|l|l|l|l|l|}
\hline 0.7 & 0.309905 & 0.309941 & 0.309957 & 0.310092 & 0.310632 \\
\hline 0.8 & 0.227817 & 0.227845 & 0.227858 & 0.227961 & 0.228375 \\
\hline 0.9 & 0.120687 & 0.120702 & 0.120709 & 0.120765 & 0.120990 \\
\hline$L_{2} \times 10^{3}$ & & 0.027891 & 0.040351 & 0.144068 & 0.559026 \\
\hline$L_{\infty} \times 10^{3}$ & & 0.039811 & 0.057680 & 0.206409 & 0.799014 \\
\hline
\end{tabular}

Tablo 2. Problem 1 için TK-LSFY ile elde edilen çözümler ile tam çözümlerin karşılaştırılması

\begin{tabular}{|l|l|l|l|l|l|}
\hline$x$ & $\begin{array}{l}\text { Tam } \\
\text { Çözüm }\end{array}$ & $N=100$ & $N=80$ & $N=40$ & $N=20$ \\
\hline 0.1 & 0.109538 & 0.109549 & 0.109553 & 0.109588 & 0.109730 \\
\hline 0.2 & 0.209792 & 0.209812 & 0.209821 & 0.209890 & 0.210170 \\
\hline 0.3 & 0.291896 & 0.291925 & 0.291937 & 0.292039 & 0.292444 \\
\hline 0.4 & 0.347924 & 0.347959 & 0.347974 & 0.348102 & 0.348612 \\
\hline 0.5 & 0.371577 & 0.371617 & 0.371634 & 0.371778 & 0.372357 \\
\hline 0.6 & 0.359046 & 0.359085 & 0.359103 & 0.359251 & 0.359844 \\
\hline 0.7 & 0.309905 & 0.309941 & 0.309957 & 0.310092 & 0.310631 \\
\hline 0.8 & 0.227817 & 0.227844 & 0.227857 & 0.227960 & 0.228374 \\
\hline 0.9 & 0.120687 & 0.120701 & 0.120708 & 0.120764 & 0.120990 \\
\hline$L_{2} \times 10^{3}$ & & 0.028043 & 0.040500 & 0.144210 & 0.559165 \\
\hline$L_{\infty} \times 10^{3}$ & & 0.039792 & 0.057645 & 0.206337 & 0.799083 \\
\hline
\end{tabular}

Tablo 3 ile $v=0.1, N=80$ ve $k=10^{-4}$ değerleri için $\quad t=0.4,0.6,0.8,1$ zamanlarında literatürdeki bazı yöntemlerle (Gülsu ve Öziş, 2005; Gülsu, 2006; İnan ve Bahadır, 2013a; Kutluay vd., 2004; Salkuyeh ve Sharafeh, 2009) elde edilen sayısal çözümler ve tam çözümlerin bu çalışmada elde edilen sayısal çözümler ile karşılaştırması sunulmuştur. Tablodan sunulan yöntemlerin daha iyi sonuçlar verdiği görülmektedir. Tablo 4 ile $v=0.005,0.01, \quad N=80 \quad$ ve $k=10^{-4}$ değerleri için K-LSFY ve TK-LSFY ile elde edilen sayısal çözümler sunulmuştur. Tablo 5 ile $v=3,5, N=80$ ve $k=10^{-4}$ değerleri için K-LSFY ve TK-LSFY ile elde edilen sayısal çözümler sunulmuştur. Tablolardan sunulan yöntemlerle elde edilen sonuçların tam çözümlere oldukça yakın olduğu görülmektedir.

Tablo 3. $v=0.1, N=80$ ve $k=10^{-4}$ değerleri için farklı zamanlarda Problem 1 için elde edilen sonuçların karşılaştırılması

\begin{tabular}{|l|l|l|l|l|l|l|l|l|l|l|}
\hline$x$ & $t$ & $\begin{array}{l}\text { Tam } \\
\text { Çözüm }\end{array}$ & K-LSFY & $\begin{array}{l}\text { TK- } \\
\text { LSFY }\end{array}$ & $\begin{array}{l}\text { (İnan ve } \\
\text { Bahadır, } \\
\text { 2013a) }\end{array}$ & $\begin{array}{l}\text { (İnan ve } \\
\text { Bahadır, } \\
\text { 2013a) }\end{array}$ & $\begin{array}{l}\text { (Salkuyeh } \\
\text { ve } \\
\text { Sharafeh, } \\
\text { 2009) }\end{array}$ & $\begin{array}{l}\text { (Gülsu, } \\
\text { 2006) }\end{array}$ & $\begin{array}{l}\text { (Gülsu } \\
\text { ve Öziş, } \\
\text { 2005) }\end{array}$ & $\begin{array}{l}\text { (Kutluay } \\
\text { vd., } \\
\text { 2004) }\end{array}$ \\
\hline \multirow{3}{*}{0.25} & 0.4 & 0.308894 & 0.308911 & 0.308937 & 0.308936 & 0.308962 & 0.30415 & 0.308776 & 0.317062 & 0.31215 \\
\cline { 2 - 11 } & 0.6 & 0.240739 & 0.240754 & 0.240774 & 0.240775 & 0.240795 & 0.23629 & 0.240654 & 0.248472 & 0.24360 \\
\cline { 2 - 10 } & 0.8 & 0.195676 & 0.195692 & 0.195708 & 0.195709 & 0.195725 & 0.19150 & 0.195579 & 0.202953 & 0.19815 \\
\hline
\end{tabular}




\begin{tabular}{|l|l|l|l|l|l|l|l|l|l|l|}
\hline & 1.0 & 0.162565 & 0.162585 & 0.162597 & 0.162599 & 0.162612 & 0.15861 & 0.162513 & 0.169527 & 0.16473 \\
\hline \multirow{3}{*}{0.50} & 0.4 & 0.569632 & 0.569684 & 0.569719 & 0.569727 & 0.569762 & 0.56711 & 0.569527 & 0.583408 & 0.57293 \\
\cline { 2 - 10 } & 0.6 & 0.447206 & 0.447268 & 0.447299 & 0.447307 & 0.447337 & 0.44360 & 0.447117 & 0.461714 & 0.40588 \\
\cline { 2 - 11 } & 0.8 & 0.359236 & 0.359309 & 0.359334 & 0.359343 & 0.359368 & 0.35486 & 0.359161 & 0.373800 & 0.36286 \\
\cline { 2 - 11 } & 1.0 & 0.291916 & 0.291995 & 0.292015 & 0.292026 & 0.292046 & 0.28710 & 0.291843 & 0.306184 & 0.29532 \\
\hline \multirow{3}{*}{0.75} & 0.4 & 0.625438 & 0.625619 & 0.625636 & 0.625659 & 0.625676 & 0.61874 & 0.625341 & 0.638847 & 0.63038 \\
\cline { 2 - 10 } & 0.6 & 0.487215 & 0.487447 & 0.487465 & 0.487495 & 0.487513 & 0.47855 & 0.487089 & 0.506429 & 0.49268 \\
\cline { 2 - 10 } & 0.8 & 0.373922 & 0.374141 & 0.374157 & 0.374187 & 0.374203 & 0.36467 & 0.373827 & 0.393565 & 0.37912 \\
\cline { 2 - 10 } & 1.0 & 0.287474 & 0.287659 & 0.287673 & 0.287700 & 0.287714 & 0.27860 & 0.029726 & 0.305862 & 0.03038 \\
\hline
\end{tabular}

Tablo 4. Problem 1 için $v=0.01, v=0.005, N=80$ ve $k=10^{-4}$ değerleri için elde edilen sayısal çözümlerin tam çözümlerle karşılaştırılması

\begin{tabular}{|l|l|l|l|l|l|l|l|}
\hline \multirow{3}{*}{$x$} & \multirow{2}{*}{$t$} & \multicolumn{3}{|c|}{$v=0.01$} & \multicolumn{3}{c|}{$v=0.005$} \\
\cline { 3 - 8 } & $\begin{array}{l}\text { Tam } \\
\text { Çözüm }\end{array}$ & K-LSFY & $\begin{array}{l}\text { TK- } \\
\text { LSFY }\end{array}$ & $\begin{array}{l}\text { Tam } \\
\text { Çözüm }\end{array}$ & K-LSFY & $\begin{array}{l}\text { TK- } \\
\text { LSFY }\end{array}$ \\
\hline \multirow{5}{*}{0.25} & 3 & 0.075114 & 0.075115 & 0.075120 & 0.075227 & 0.075227 & 0.075232 \\
\cline { 2 - 8 } & 5 & 0.046917 & 0.046918 & 0.046920 & 0.046963 & 0.046964 & 0.046966 \\
\cline { 2 - 8 } & 10 & 0.024069 & 0.024071 & 0.024072 & 0.024217 & 0.024217 & 0.024218 \\
\cline { 2 - 8 } & 15 & 0.015375 & 0.015379 & 0.015379 & 0.016308 & 0.016308 & 0.016308 \\
\hline \multirow{4}{*}{0.50} & 3 & 0.150179 & 0.150180 & 0.150190 & 0.150408 & 0.150409 & 0.150420 \\
\cline { 2 - 8 } & 5 & 0.093796 & 0.093798 & 0.093803 & 0.093920 & 0.093920 & 0.093925 \\
\cline { 2 - 8 } & 10 & 0.046890 & 0.046901 & 0.046903 & 0.048421 & 0.048422 & 0.048424 \\
\cline { 2 - 8 } & 15 & 0.027810 & 0.027823 & 0.027824 & 0.032439 & 0.032442 & 0.032443 \\
\hline \multirow{3}{*}{0.75} & 3 & 0.224811 & 0.224842 & 0.224857 & 0.225498 & 0.225499 & 0.225514 \\
\cline { 2 - 8 } & 5 & 0.137307 & 0.137389 & 0.137396 & 0.140832 & 0.140837 & 0.140845 \\
\cline { 2 - 8 } & 10 & 0.056804 & 0.056863 & 0.056866 & 0.071134 & 0.071173 & 0.071176 \\
\cline { 2 - 8 } & 15 & 0.027569 & 0.027596 & 0.027597 & 0.044133 & 0.044174 & 0.044175 \\
\hline
\end{tabular}

Tablo 5. Problem 1 için $v=3, v=5, N=80$ ve $k=10^{-4}$ değerleri için elde edilen sayısal çözümlerin tam çözümlerle karşılaştırılması

\begin{tabular}{|l|l|l|l|l|l|l|l|}
\hline \multirow{3}{*}{$x$} & \multirow{2}{*}{$t$} & \multicolumn{3}{|c|}{$v=3$} & \multicolumn{3}{c|}{$v=5$} \\
\cline { 3 - 8 } & & $\begin{array}{l}\text { Tam } \\
\text { Çözüm }\end{array}$ & K-LSFY & $\begin{array}{l}\text { TK- } \\
\text { LSFY }\end{array}$ & $\begin{array}{l}\text { Tam } \\
\text { Çözüm }\end{array}$ & K-LSFY & $\begin{array}{l}\text { TK- } \\
\text { LSFY }\end{array}$ \\
\hline \multirow{4}{*}{0.25} & 0.01 & 0.519350 & 0.519454 & 0.519474 & 0.427964 & 0.428181 & 0.428181 \\
\cline { 2 - 8 } & 0.05 & 0.159546 & 0.159767 & 0.159771 & 0.059845 & 0.060113 & 0.060113 \\
\cline { 2 - 8 } & 0.1 & 0.036526 & 0.036656 & 0.036656 & 0.005084 & 0.005139 & 0.005139 \\
\hline \multirow{4}{*}{0.50} & 0.01 & 0.743596 & 0.743693 & 0.743694 & 0.610441 & 0.610703 & 0.610703 \\
\cline { 2 - 8 } & 0.05 & 0.227458 & 0.227776 & 0.227777 & 0.084794 & 0.085174 & 0.085174 \\
\cline { 2 - 8 } & 0.1 & 0.051755 & 0.051940 & 0.051940 & 0.007191 & 0.007268 & 0.007268 \\
\hline \multirow{3}{*}{0.75} & 0.01 & 0.532462 & 0.532541 & 0.532523 & 0.435405 & 0.435618 & 0.435618 \\
\cline { 2 - 8 } & 0.05 & 0.162149 & 0.162385 & 0.162382 & 0.060072 & 0.060344 & 0.060343 \\
\cline { 2 - 8 } & 0.1 & 0.036667 & 0.036799 & 0.036799 & 0.005086 & 0.005140 & 0.005140 \\
\hline
\end{tabular}


Tablo 6-10, Problem 2 için sunulan yöntemler ile elde edilen sayısal sonuçları göstermektedir. $v=1, k=10^{-5}$ ve $N^{\prime}$ nin farklı değerleri için $t=0.1$ zamanında $\mathrm{K}$ LSFY kullanılarak elde edilen sayısal sonuçlar Tablo 6 ile sunulmuştur. $v=1$, $k=10^{-5}$ ve $N$, nin farklı değerleri için $t=0.1$ zamanında TK-LSFY kullanılarak elde edilen sayısal sonuçlar Tablo 7 ile sunulmuştur. Tablo 6 ve Tablo 7 den $N$, nin değeri büyüdükçe yani konum için bölüntü sayısı $\operatorname{arttıkça~} L_{2}$ ve $L_{\infty}$ hata normlarının değerlerinin azaldığ görülmektedir.

Tablo 6. Problem 2 için K-LSFY ile elde edilen çözümler ile tam çözümlerin karşılaştırılması

\begin{tabular}{|l|l|l|l|l|l|}
\hline$x$ & $\begin{array}{l}\text { Tam } \\
\text { Çözüm }\end{array}$ & $N=100$ & $N=80$ & $N=40$ & $N=20$ \\
\hline 0.1 & 0.112892 & 0.112902 & 0.112907 & 0.112943 & 0.113089 \\
\hline 0.2 & 0.216252 & 0.216272 & 0.216280 & 0.216352 & 0.216639 \\
\hline 0.3 & 0.300966 & 0.300994 & 0.301007 & 0.301111 & 0.301527 \\
\hline 0.4 & 0.358863 & 0.358898 & 0.358914 & 0.359045 & 0.359569 \\
\hline 0.5 & 0.383422 & 0.383462 & 0.383480 & 0.383629 & 0.384224 \\
\hline 0.6 & 0.370658 & 0.370699 & 0.370717 & 0.370870 & 0.371482 \\
\hline 0.7 & 0.320066 & 0.320103 & 0.320120 & 0.320260 & 0.320819 \\
\hline 0.8 & 0.235371 & 0.235400 & 0.235413 & 0.235520 & 0.235950 \\
\hline 0.9 & 0.124718 & 0.124734 & 0.124741 & 0.124800 & 0.125034 \\
\hline$L_{2} \times 10^{3}$ & & 0.028702 & 0.041574 & 0.148686 & 0.576756 \\
\hline$L_{\infty} \times 10^{3}$ & & 0.040957 & 0.059417 & 0.213016 & 0.824419 \\
\hline
\end{tabular}

Tablo 7. Problem 2 için TK-LSFY ile elde edilen çözümler ile tam çözümlerin karşılaştırılması

\begin{tabular}{|l|l|l|l|l|l|}
\hline$x$ & $\begin{array}{l}\text { Tam } \\
\text { Çözüm }\end{array}$ & $N=100$ & $N=80$ & $N=40$ & $N=20$ \\
\hline 0.1 & 0.112892 & 0.112903 & 0.112907 & 0.112944 & 0.113089 \\
\hline 0.2 & 0.216252 & 0.216273 & 0.216281 & 0.216353 & 0.216640 \\
\hline 0.3 & 0.300966 & 0.300995 & 0.301008 & 0.301112 & 0.301528 \\
\hline 0.4 & 0.358863 & 0.358899 & 0.358915 & 0.359046 & 0.359570 \\
\hline 0.5 & 0.383422 & 0.383463 & 0.383480 & 0.383629 & 0.384225 \\
\hline 0.6 & 0.370658 & 0.370698 & 0.370717 & 0.370870 & 0.371482 \\
\hline 0.7 & 0.320066 & 0.320102 & 0.320119 & 0.320259 & 0.320818 \\
\hline 0.8 & 0.235371 & 0.235399 & 0.235412 & 0.235520 & 0.235950 \\
\hline 0.9 & 0.124718 & 0.124733 & 0.124740 & 0.124799 & 0.125034 \\
\hline$L_{2} \times 10^{3}$ & & 0.028861 & 0.041729 & 0.148834 & 0.576899 \\
\hline$L_{\infty} \times 10^{3}$ & & 0.040917 & 0.059356 & 0.212937 & 0.824183 \\
\hline
\end{tabular}

Tablo 8 ile $v=0.1, N=80$ ve $k=10^{-4}$ değerleri için $\quad t=0.4,0.6,0.8,1$ zamanlarında literatürdeki bazı yöntemlerle (Gülsu ve Öziş, 2005; Gülsu, 
2006; İnan ve Bahadır, 2013a; Kutluay vd., 2004; Salkuyeh ve Sharafeh, 2009) elde edilen sayısal çözümler ve tam çözümlerin bu çalışmada elde edilen sayısal çözümler ile karşılaştırması sunulmuştur. Tablodan sunulan yöntemlerin daha iyi sonuçlar verdiği görülmektedir. Tablo 9 ile $v=0.005,0.01, \quad N=80 \quad$ ve $\quad k=10^{-4}$ değerleri için K-LSFY ve TK-LSFY ile elde edilen sayısal çözümler sunulmuştur. Tablo 10 ile $v=3,5, N=80$ ve $k=10^{-4}$ değerleri için K-LSFY ve TK-LSFY ile elde edilen sayısal çözümler sunulmuştur. Tablolardan sunulan yöntemlerle elde edilen sonuçların tam çözümlere oldukça yakın olduğu görülmektedir.

Tablo 8. $v=0.1, N=80$ ve $k=10^{-4}$ değerleri için farklı zamanlarda Problem 2 için elde edilen sonuçların karşılaştırılması

\begin{tabular}{|c|c|c|c|c|c|c|c|c|c|c|}
\hline \multirow[b]{2}{*}{$x$} & \multirow[b]{2}{*}{$t$} & \multirow[b]{2}{*}{$\begin{array}{l}\text { Tam } \\
\text { Çözüm }\end{array}$} & \multicolumn{3}{|c|}{$k=10^{-5}$} & \multicolumn{5}{|c|}{$k=10^{-4}$} \\
\hline & & & $\begin{array}{l}\text { (Gülsu } \\
\text { ve Öziş, } \\
2005)\end{array}$ & $\begin{array}{l}\text { (Gülsu, } \\
\text { 2006) }\end{array}$ & $\begin{array}{l}\text { (Kutluay } \\
\text { vd., } \\
2004 \text { ) }\end{array}$ & $\begin{array}{l}\text { (Salkuyeh } \\
\text { ve } \\
\text { Sharafeh, } \\
2009 \text { ) }\end{array}$ & $\begin{array}{l}\text { (İnan ve } \\
\text { Bahadır, } \\
2013 a \text { ) }\end{array}$ & $\begin{array}{l}\text { (İnan ve } \\
\text { Bahadır, } \\
2013 a \text { ) }\end{array}$ & K-LSFY & $\begin{array}{l}\text { TK- } \\
\text { LSFY }\end{array}$ \\
\hline \multirow{4}{*}{0.25} & 0.4 & 0.317523 & 0.306529 & 0.317399 & 0.32091 & 0.31247 & 0.317567 & 0.317595 & 0.317539 & 0.317567 \\
\hline & 0.6 & 0.246138 & 0.236051 & 0.246058 & 0.24910 & 0.24148 & 0.246175 & 0.246196 & 0.246153 & 0.246174 \\
\hline & 0.8 & 0.199555 & 0.190181 & 0.199437 & 0.20211 & 0.19524 & 0.199589 & 0.199606 & 0.199571 & 0.199588 \\
\hline & 1.0 & 0.165599 & 0.156646 & 0.165529 & 0.16782 & 0.16153 & 0.165633 & 0.165647 & 0.162585 & 0.165631 \\
\hline \multirow{4}{*}{0.50} & 0.4 & 0.584537 & 0.565994 & 0.584429 & 0.58788 & 0.58176 & 0.584627 & 0.584664 & 0.584582 & 0.584618 \\
\hline & 0.6 & 0.457976 & 0.438926 & 0.457888 & 0.46174 & 0.45414 & 0.458077 & 0.458110 & 0.458037 & 0.458069 \\
\hline & 0.8 & 0.367398 & 0.348328 & 0.367320 & 0.37111 & 0.36283 & 0.367507 & 0.367533 & 0.367470 & 0.367497 \\
\hline & 1.0 & 0.298343 & 0.280038 & 0.298271 & 0.30183 & 0.29336 & 0.298455 & 0.298476 & 0.291995 & 0.298443 \\
\hline \multirow{4}{*}{0.75} & 0.4 & 0.645616 & 0.626990 & 0.645527 & 0.65054 & 0.63858 & 0.645850 & 0.645866 & 0.645810 & 0.645826 \\
\hline & 0.6 & 0.502676 & 0.477908 & 0.502564 & 0.50825 & 0.49362 & 0.502969 & 0.502987 & 0.502919 & 0.502937 \\
\hline & 0.8 & 0.385336 & 0.360630 & 0.385232 & 0.39068 & 0.37570 & 0.385613 & 0.385630 & 0.385564 & 0.385581 \\
\hline & 1.0 & 0.295857 & 0.272623 & 0.295779 & 0.30057 & 0.28663 & 0.296092 & 0.296106 & 0.287659 & 0.296064 \\
\hline
\end{tabular}

Tablo 9. Problem 2 için $v=0.01, v=0.005, N=80$ ve $k=10^{-4}$ değerleri için elde edilen sayısal çözümlerin tam çözümlerle karşılaştırılması

\begin{tabular}{|l|l|l|l|l|l|l|l|}
\hline \multirow{3}{*}{$x$} & \multirow{4}{*}{$t$} & \multicolumn{3}{|c|}{$v=0.01$} & \multicolumn{3}{c|}{$v=0.005$} \\
\cline { 3 - 8 } & & $\begin{array}{l}\text { Tam } \\
\text { Çözüm }\end{array}$ & K-LSFY & $\begin{array}{l}\text { TK- } \\
\text { LSFY }\end{array}$ & K-LSFY & $\begin{array}{l}\text { TK- } \\
\text { LSFY }\end{array}$ & $\begin{array}{l}\text { Tam } \\
\text { Çözüm }\end{array}$ \\
\hline \multirow{5}{*}{0.25} & 3 & 0.076134 & 0.076135 & 0.076140 & 0.076392 & 0.076398 & 0.076391 \\
\cline { 2 - 8 } & 5 & 0.047312 & 0.047312 & 0.047315 & 0.047415 & 0.047418 & 0.047415 \\
\cline { 2 - 8 } & 10 & 0.024177 & 0.024179 & 0.024179 & 0.024336 & 0.024337 & 0.024336 \\
\cline { 2 - 8 } & 15 & 0.015432 & 0.015436 & 0.015436 & 0.016362 & 0.016362 & 0.016362 \\
\hline \multirow{3}{*}{0.50} & 3 & 0.152180 & 0.152181 & 0.152192 & 0.152681 & 0.152692 & 0.152679 \\
\cline { 2 - 8 } & 5 & 0.094582 & 0.094584 & 0.094589 & 0.094815 & 0.094820 & 0.094814 \\
\cline { 2 - 8 } & 10 & 0.047122 & 0.047134 & 0.047136 & 0.048661 & 0.048662 & 0.048660 \\
\cline { 2 - 8 } & 15 & 0.027934 & 0.027947 & 0.027948 & 0.032553 & 0.032554 & 0.032550 \\
\hline & 3 & 0.227743 & 0.227772 & 0.227789 & 0.228769 & 0.228786 & 0.228768 \\
\hline
\end{tabular}




\begin{tabular}{|l|l|l|l|l|l|l|l|}
\hline 0.75 & 5 & 0.138568 & 0.138649 & 0.138657 & 0.142160 & 0.142168 & 0.142154 \\
\cline { 2 - 8 } & 10 & 0.057197 & 0.057257 & 0.057259 & 0.071557 & 0.071559 & 0.071517 \\
\cline { 2 - 8 } & 15 & 0.027733 & 0.027760 & 0.027761 & 0.044370 & 0.044371 & 0.044328 \\
\hline
\end{tabular}

Tablo 10. Problem 2 için $v=3, v=5, N=80$ ve $k=10^{-4}$ değerleri için elde edilen sayısal çözümlerin tam çözümlerle karşılaştırılması

\begin{tabular}{|l|l|l|l|l|l|l|l|}
\hline \multirow{2}{*}{$x$} & \multirow{t}{*}{\begin{tabular}{l}
$t$ \\
\cline { 3 - 8 }
\end{tabular}} & $\begin{array}{l}\text { Tam } \\
\text { Çözüm }\end{array}$ & K-LSFY & $\begin{array}{l}\text { TK- } \\
\text { LSFY }\end{array}$ & $\begin{array}{l}\text { Tam } \\
\text { Çözüm }\end{array}$ & K-LSFY & $\begin{array}{l}\text { TK- } \\
\text { LSFY }\end{array}$ \\
\hline \multirow{4}{*}{0.25} & 0.01 & 0.537833 & 0.538000 & 0.538022 & 0.441930 & 0.442182 & 0.442182 \\
\cline { 2 - 8 } & 0.05 & 0.164616 & 0.164841 & 0.164845 & 0.061759 & 0.062033 & 0.062033 \\
\cline { 2 - 8 } & 0.1 & 0.037693 & 0.037826 & 0.037827 & 0.005247 & 0.005303 & 0.005303 \\
\hline \multirow{4}{*}{0.50} & 0.01 & 0.764770 & 0.764784 & 0.764785 & 0.629552 & 0.629779 & 0.629779 \\
\cline { 2 - 8 } & 0.05 & 0.234743 & 0.235068 & 0.235069 & 0.087511 & 0.087900 & 0.087900 \\
\cline { 2 - 8 } & 0.1 & 0.053413 & 0.053602 & 0.053602 & 0.007421 & 0.007501 & 0.007501 \\
\hline \multirow{3}{*}{0.75} & 0.01 & 0.551442 & 0.551591 & 0.551571 & 0.449746 & 0.449995 & 0.449995 \\
\cline { 2 - 8 } & 0.05 & 0.167385 & 0.167626 & 0.167622 & 0.062001 & 0.062279 & 0.062278 \\
\cline { 2 - 8 } & 0.1 & 0.037844 & 0.037979 & 0.037979 & 0.005249 & 0.005305 & 0.005305 \\
\hline
\end{tabular}

\section{Sonuç ve Tartışma}

Bu çalışmada, Burgers denklemini çözmek için iki farklı logaritmik sonlu fark yöntemi tanımland1. Von-Neumann kararlılık yöntemi kullanılarak sunulan logaritmik sonlu fark yöntemlerinin şartsız kararlı olduğu gösterildi. İki farklı model problem için sayısal çözümler verildi. Sonuçlar, hem kapalı hem de tamamen kapalı logaritmik sonlu fark yönteminin, tek boyutlu Burgers denkleminin sayısal çözümü için yüksek doğruluk sağladığını göstermektedir.

\section{Kaynaklar}

Aksan, E. N., Özdeş, A. 2004. "A numerical solution of Burgers' equation", Applied Mathematics and Computation, 156, 395-402.
Aksan, E. N. 2005. "A numerical solution of Burgers' equation by finite element method constructed on the method of discretization in time", Applied Mathematics and Computation, 170, 895904.

Bateman, H. 1915. "Some recent researches in motion of fluids", Monthly Weather Review, 43, 163-170.

Burgers, J. M. 1939. "Mathematical examples illustrating relations occuring in the theory of turbulent fluid motion", Transactions of the Royal Netherlands Academy of Science (Amsterdam), 17, 1153.

Çelikten, G., Göksu, A. \& Yagub, G. 2017. "Explicit Logarithmic Finite Difference Schemes For Numerical Solution of Burgers Equation", European International Journal of Science and Technology, 6(5), 57-67. 
Gülsu, M., Öziş, T. 2005. "Numerical solution of Burgers' equation with restrictive Taylor Approximation", Applied Mathematics and Computation, 171, 11921200.

Gülsu, M. 2006. "A finite difference approach for solution of Burgers' equation", Applied Mathematics and Computation, 175, 1245-1255.

İnan, B., Bahadır, A. R. 2013a. "Numerical solution of the one-dimensional Burgers' equation: Implicit and fully implicit exponential finite difference methods", PRAMANA journal of physics, 81(4), 547556.

İnan, B., Bahadır, A. R. 2013b. "An explicit exponential finite difference method for the Burgers' equation", European International Journal of Science and Technology, 2(10), 61-72.

Jiwari, R. 2012. "A Haar wavelet quasilinearization approach for numerical simulation of Burgers' equation", Computer Physics Communications, 183(11), 2413-2423.

Korkmaz, A., Dag, I. 2011. "Polynomial based differential quadrature method for numerical solution of nonlinear Burgers' equation", Journal of the Franklin Institute, 348(10), 2863-2875.

Kutluay, S., Esen, A. \& Dag, I. 2004. 'Numerical solutions of the Burgers' equation by the least-squares quadratic Bspline finite element method", Journal of Computational and Applied Mathematics, 167, 21-33.

Mittal, R. C., Jain, R. K. 2012. "Numerical solutions of nonlinear Burgers' equation with modified cubic Bsplines collocation method" Applied Mathematics and Computation, 218(15), 7839-7855.

Öziş, T., Aksan, E. N. and Özdeş, A. 2003. "A finite element approach for solution of Burgers' equation", Applied Mathematics and Computation, 139, 417-428.
Salkuyeh, D. K., Sharafeh, F. S. 2009. “On the numerical solution of the Burgers's equation", International Journal of Computer Mathematics, 86, 1334-1344.

Zhang, P. G., Wang, J. P. 2012. “A predictor-corrector compact finite difference scheme for Burgers' equation", Applied Mathematics and Computation, 219(3), 892-898. 